PROCEEDINGS OF THE

AMERICAN MATHEMATICAL SOCIETY

Volume 132, Number 9, Pages 2603-2611

S 0002-9939(04)06914-X

Article electronically published on April 21, 2004

\title{
SKEW EXACTNESS PERTURBATION
}

\author{
ROBIN HARTE AND DAVID LARSON
}

(Communicated by Joseph A. Ball)

\begin{abstract}
We offer a perturbation theory for finite ascent and descent properties of bounded operators.
\end{abstract}

There are various degrees of "skew exactness" (11]; [8], (10.9.0.1), (10.9.0.2)) between compatible pairs of operators, bounded and linear between normed spaces:

1. Definition. Suppose $T: X \rightarrow Y$ and $S: Y \rightarrow Z$ are bounded and linear between normed spaces. Then we may classify the pair $(S, T)$ as left skew exact if there is inclusion

$$
S^{-1}(0) \cap T(X)=\{0\},
$$

strongly left skew exact if there is $k>0$ for which

$$
\|T(\cdot)\| \leq k\|S T(\cdot)\|,
$$

and splitting left skew exact if there is $R \in B L(Z, Y)$ for which

$$
T=R S T \text {. }
$$

Also we may classify the pair $(S, T)$ as right skew exact if there is inclusion

$$
S^{-1}(0)+T(X)=Y,
$$

strongly right skew exact if there is $k>0$ for which: for every $y \in Y$ there is $x \in X$ for which

$$
S y=S T x \text { with }\|x\| \leq k\|y\|,
$$

and splitting right skew exact if there is $R \in B L(Y, X)$ for which

$$
S=S T R \text {. }
$$

It is easy to see that

2. Theorem. In the notation of Definition 1, there exist implications

$$
(1.3) \Longrightarrow(1.2) \Longrightarrow(1.1)
$$

and

$$
(1.6) \Longrightarrow(1.5) \Longrightarrow(1.4)
$$

Received by the editors January 28, 2002 and, in revised form, May 24, 2002.

2000 Mathematics Subject Classification. Primary 47A10.

The first author was partially supported by Enterprise Ireland grant number IC/2001/027.

(C)2004 American Mathematical Society 
Proof. Most of this holds slightly more generally ([8], Theorems 10.1.2, 10.1.4), with a general operator $R^{\prime}: X \rightarrow Z$ in place of the product $S T$. Note (cf. [4], (6.1)) that (1.1) holds iff

$$
(S T)^{-1}(0) \subseteq T^{-1}(0)
$$

and that (1.4) holds iff

$$
S(Y) \subseteq S T(X) .
$$

For Hilbert spaces $X, Y, Z$ there exist ( 8 , Theorem 10.8.1) implications (1.2) $\Longrightarrow$ (1.3) and (1.5) $\Longrightarrow(1.6)$.

A slightly stronger version of condition (1.1) asks that

$$
S^{-1}(0) \cap \operatorname{cl} T(X)=\{0\},
$$

which says that the operator $K_{M} J_{N}$ is one-to-one, where (cf. Yang [17; 9]) $K_{M}$ : $Y \rightarrow Y / M$ and $J_{N}: N \rightarrow Y$ are the natural quotient and injection induced by the subspaces $M=\operatorname{cl} T X$ and $N=S^{-1}(0)$. Stronger again is the condition that there be $k>0$ for which there is implication

$$
y \in S^{-1}(0) \Longrightarrow\|y\| \leq k \operatorname{dist}(y, T(X)),
$$

which says that the same operator $K_{M} J_{N}$ is bounded below. Evidently

$$
(1.2) \Longrightarrow(2.6) \Longrightarrow(2.5) \Longrightarrow(1.1):
$$

if $k>0$ satisfies (1.2) and if $S y=0$, then

$$
\|y\| \leq\|y-T x\|+\|T x\| \leq\|y-T x\|+k\|S(T x-y)\| \leq(1+k\|S\|)\|y-T x\| .
$$

Condition (2.6), with $k=1$, has been noticed by Anderson 11, who describes it by calling $T(X)$ orthogonal to $S^{-1}(0)$; we will prefer to say that $S^{-1}(0)$ is orthogonal to $T(X)$. Turnsek [16] has observed that it holds for certain operators on Banach algebras:

3. Theorem. If $S \in B L(Y, Y)$, then (2.6) holds with $k=1$ for $(S, S)$ provided

$$
\|I-S\| \leq 1 \text {. }
$$

Proof. Following the argument of Turnsek ([16], Theorem 1.1), write

$$
S=I-U \text { and } V_{n}=I+U+\ldots+U^{n},
$$

so that

$$
S V_{n}=I-U^{n+1}=V_{n} S
$$

We have

$$
S y=0 \Longrightarrow(n+1) y=V_{n} y=\left(I-U^{n+1}\right) x+V_{n}(y-S x),
$$

and hence

$$
\|y\| \leq \frac{2}{n+1}\|x\|+\|y-S x\|
$$

now let $n \rightarrow \infty$. 
Alternatively, Theorem 3 is an application of Sinclair's Theorem ([15], Proposition 1; [3], Corollary 1), since here 0 is not in the interior of the "numerical range" of $S$. The argument of Theorem 3 suggests - wrongly - that we are using a weakened version of condition (1.3): we call the pair $(S, T)$ almost splitting left skew exact if there are $\left(R_{n}\right)$ in $B L(Z, Y)$ with

$$
\left\|T-R_{n} S T\right\| \rightarrow 0 \text { and } \sup _{n}\left\|R_{n}\right\|<\infty,
$$

and almost splitting right skew exact if instead $\left(R_{n}\right)$ in $B L(Y, X)$ with

$$
\left\|S-S T R_{n}\right\| \rightarrow 0 \text { and } \sup _{n}\left\|R_{n}\right\|<\infty .
$$

Also call $(S, T)$ almost strongly right skew exact if there is $k>0$ for which: for every $y \in Y$ there is $\left(x_{n}\right)$ in $X$ for which

$$
\left\|S y-S T x_{n}\right\| \rightarrow 0 \text { with } \sup _{n}\left\|x_{n}\right\| \leq k\|y\| .
$$

Evidently (cf. [11, Theorem 10.1.2)

$$
(1.3) \Longrightarrow(3.3) \Longrightarrow(1.2)
$$

and

$$
(1.6) \Longrightarrow(3.4) \Longrightarrow(3.5) \text {; }
$$

thus (3.3) implies (2.6). We do not, however, derive (3.3) for $(S, S)$ from condition (3.1). We also remark that, whenever the space $Z$ is complete, there is the implication

$$
(1.4) \Longrightarrow(3.5) \text {. }
$$

This (2]; [5], Theorem 1.1; 8], Theorem 10.5.5) uses Baire's theorem.

Under certain circumstances the "left" and "right" skew exactnesses are equivalent. We begin (cf. [4, Lemma 6.2) by extending the finite ascent/descent characterizations:

4. Theorem. Suppose, under the conditions of Definition 1 , that $W \subseteq X$ with $T(W) \subseteq S^{-1}(0)$, and that $V \subseteq Y$ with $T(X) \subseteq S^{-1}(V)$. Then each of the following conditions is equivalent to (1.1):

$$
\begin{gathered}
T^{\vee}: X / T^{-1}(0) \rightarrow Y / S^{-1}(0) \text { one-to-one } ; \\
S^{\wedge}: T(X) \rightarrow V \text { is one-to-one. }
\end{gathered}
$$

Also each of the following conditions is equivalent to condition (1.4):

$$
\begin{gathered}
S^{\wedge}: T(X) \rightarrow S(Y) \text { onto } ; \\
T^{\vee}: X / W \rightarrow Y / S^{-1}(0) \text { is onto } .
\end{gathered}
$$

Proof. The equivalences $(1.1) \Longleftrightarrow(4.1)$ and (1.4) (1.1) is equivalent to (4.2) with $V=Z$, and that this in turn is equivalent to (4.2) for arbitrary $V$ for which $T(X) \subseteq S^{-1} V$. The second equivalence is clear; for the first note that for arbitrary $x \in X$ there is the implication

$$
T x \in S^{-1}(0) \Longleftrightarrow S T x=0 \text {. }
$$

We also claim that (1.4) is equivalent to (4.4) with $W=\{0\}$, and that this in turn is equivalent to (4.4) for arbitrary $W$ for which $T(W) \subseteq S^{-1}(0)$. The second equivalence is clear; for the first note that for arbitrary $y \in Y$ there is the implication

$$
y \in S^{-1}(0)+T(X) \Longleftrightarrow S y \in S(T X) .
$$


If in particular $X=Y=Z$ and $S T=T S$, then (4.2) applies with $V=T(X)$, and (4.4) applies with $W=S^{-1}(0)$. We apply this in particular with $S=T^{k}$ for some $k \in \mathbf{N}$ :

5. Theorem. If $X=Y=Z$ and $S=T^{k}: Y \rightarrow Y$, with $T$ in the "commutative closure" of the invertibles, in the sense that there are $\left(R_{n}\right)$ in $B L(X, X)$ with

$$
R_{n} \in B L^{-1}(X, X), R_{n} T=T R_{n},\left\|R_{n}-T\right\| \rightarrow 0,
$$

then the following are equivalent:

$$
\begin{gathered}
(T S)^{-1}(0) \subseteq S^{-1}(0) \text { and } T(X)=\operatorname{cl} T(X), \\
S(Y) \subseteq S T(X) \text { and } T(X)=\operatorname{cl} T(X) .
\end{gathered}
$$

Proof. We recall ([6]; $[\overline{8}$, Theorem 3.5.1) that for bounded linear operators $T$ : $X \rightarrow Y$ between (possibly incomplete) normed spaces

$$
T \text { bounded below and a limit of dense range } \Longrightarrow T \text { almost open , }
$$

and hence (6]; 8$]$, Theorem 5.5.6) by duality

$$
T \text { almost open and a limit of bounded below } \Longrightarrow T \text { bounded below . }
$$

Now if $R_{n}$ commutes with $T$, then it leaves both $T(X)$ and $S^{-1}(0)$ invariant, and if $R_{n}$ is invertible, then (cf. 8), Theorem 3.11.1) its restriction $R_{n}^{\wedge}$ to $T(X)$ will be bounded below and its quotient on $Y / S^{-1}(0)$ will be onto. Thus if we assume (5.2), then by (4.1) and closed range, $T^{\vee}$ will be bounded below and the limit of onto $R_{n}^{\vee}$, therefore onto, giving (5.3). If instead we assume (5.3), then by (4.3) $S^{\wedge}$ will be onto and by closed range almost open, and the limit of bounded below $\left(R_{n}^{k}\right)^{\wedge}$, therefore bounded below, giving (5.2).

(5.2) and (5.3) are together equivalent to the condition that $T \in B L(X, X)$ is polar (8), Definition 7.5.2) in the sense that $0 \in \mathbf{C}$ is at worst a pole of the resolvent function $(z I-T)^{-1}$. If we relax the closed range condition we can still 14] get one of the implications, provided we further tighten the approximation by commuting invertible operators:

6. Theorem. Suppose that $S=T^{k}$ and that $0 \notin$ int $\sigma(T)$. If the finite descent condition (1.4) holds, then so also does the finite ascent condition (5.2), including closed range.

Proof. This is shown on Hilbert space ([14, Lemma 2.5) by Herrero, Larson and Wogen. Alternatively, since we are assuming that 0 is at worst on the boundary of the spectrum, then we can take the approximating invertible operators $R_{n}=$ $T-\lambda_{n} I$ to be scalar perturbations of the operator $T$. Now if (1.4) holds, then the quotient operator $T^{\vee}$ on $X / S^{-1}(0)$ is (4.5) onto, and the limit of operators $\left(T-\lambda_{n} I\right)^{\vee}$, which we claim are invertible. As in Theorem 5 it is clear that the quotient $\left(T-\lambda_{n} I\right)^{\vee}$ is onto: we claim it is also one-to-one. To see this recall that the operator $T-\lambda_{n} I$ is one-to-one and the restriction $\left(T-\lambda_{n} I\right)^{\wedge}=\left(-\lambda_{n} I\right)^{\wedge}$ to the subspace $T^{-1}(0)$ is onto, so that ([5], Theorem 3.11.2) the induced quotient is also one-to-one. For the closed range note that $T(X)$ now has a closed complement, and appeal to the "Lemma of Neuberger" ([8], Theorem 4.8.2). 
Theorem 6 does not reverse:

7. Example. If

$$
S=I-\lambda U \text { or } S=I-\lambda V \text { or } S=\lambda W,
$$

where $|\lambda|=1, U$ and $V$ are the forward and backward shifts on $\ell_{2}$, and $W$ the standard weight,

$$
(U x)_{1}=0,(U x)_{n+1}=x_{n} ;(V x)_{n}=x_{n+1} ;(W x)_{n}=(1 / n) x_{n},
$$

then $S$ is one-to-one and not onto, therefore of finite descent and not of finite ascent, while

$$
\|I-S\|=1 \text { so that } 0 \notin \operatorname{int} \sigma(S) .
$$

Proof. This is easily checked: note that, extended to all sequences, there is equivalence, for arbitrary $x \in X^{\mathbf{N}}$,

$$
x \in(I-\lambda U)^{-1} \Longleftrightarrow x \in(I-\bar{\lambda} V)^{-1} \Longleftrightarrow x=x_{1}\left(1, \lambda, \lambda^{2}, \ldots\right) .
$$

We need some auxiliary subspaces:

8. Definition. If $T \in B L(X, X)$ write

$$
T^{-\infty}(0)=\bigcup_{n=1}^{\infty} T^{-n}(0) \text { and } T^{\infty}(X)=\bigcap_{n=1}^{\infty} T^{n}(X)
$$

for the hyperkernel and the hyperrange of $T$, and

$$
E_{X}(T)=\sum_{\lambda \in \mathbf{C}}(T-\lambda I)^{-\infty}(0) \text { and } F_{X}(T)=\bigcap_{\lambda \in \mathbf{C}}(T-\lambda I)^{\infty}(X) .
$$

Each of the subspaces in Definition 8 is linear, not necessarily closed, and hyperinvariant under $T$. We recall that $T \in B L(X, X)$ is called algebraic if there is a nontrivial polynomial $0 \neq p \in$ Poly for which

$$
p(T)=0 ;
$$

more generally $T$ is said to be locally algebraic if

$$
X=\bigcup\left\{p(T)^{-1}(0): 0 \neq p \in \text { Poly }\right\} .
$$

For the record

9. Theorem. If $T \in B L(X, X)$ for a Banach space $X$, then

$$
T \text { locally algebraic } \Longrightarrow T \text { algebraic. }
$$

Necessary and sufficient for $T$ to have finite descent is that

$$
E_{X}(T)+T(X)=X .
$$

Proof. The first part of this is known as Kaplansky's Lemma; the proof [10], [12] is a combination of Baire's theorem and the Euclidean algorithm for polynomials. The Euclidean algorithm also gives equality

$$
E_{X}(T)=\bigcup\left\{p(T)^{-1}(0): 0 \neq p \in \operatorname{Poly}\right\}=\{x \in X: \operatorname{dim} \operatorname{Poly}(T) x<\infty\},
$$

and dually

$$
F_{X}(T)=\bigcap\{p(T)(X): 0 \neq p \in \text { Poly }\}
$$


Then again with a combination of Baire's theorem and the Euclidean algorithm, if $T \in B L(X, X)$ there is (14], Lemma 2.4) $k \in \mathbf{N}$ for which

$$
E_{X}(T)+T(X)=T^{-\infty}(0)+T(X)=T^{-k}(0)+T(X) .
$$

Dually, using the Euclidean algorithm, we get half way:

$$
F_{X}(T) \cap T^{-1}(0)=T^{\infty}(X) \cap T^{-1}(0) .
$$

For the essence of a possible spectral mapping theorem (cf. [11]), we have

10. Theorem. If $S, T \in B L(X, X)$ satisfy $S T=T S$ and either

$$
S \in\left\{T^{k}: k \in \mathbf{N}\right\}
$$

or

$$
V S-T U=I \text { with }\{U, V\} \subseteq \operatorname{comm}(S, T),
$$

then there is the equivalence

$$
\text { ST of finite ascent } \Longleftrightarrow S, T \text { of finite ascent },
$$

and the equivalence

$$
\text { ST of finite descent } \Longleftrightarrow S, T \text { of finite descent . }
$$

Proof. The backward implications are easy (8], Theorem 7.9.2): if $S$ and $T$ commute and satisfy $S^{-k}(0)=S^{-k-1}(0)$ and $T^{-k}(0)=T^{-k-1}(0)$, then

$$
\begin{aligned}
(S T)^{-k}(0) & =S^{-k} T^{-k}(0)=S^{-k} T^{-k-1}(0)=T^{-k-1} S^{-k}(0) \\
& =T^{-k-1} S^{-k-1}(0)=(S T)^{-k-1}(0) .
\end{aligned}
$$

If instead $S T=T S$ with $S^{k} X=S^{k+1} X$ and $T^{k} X=T^{k+1} X$, then

$$
(S T)^{k} X=S^{k} T^{k}(X)=S^{k} T^{k+1} X=T^{k+1} S^{k} X=T^{k+1} S^{k+1} X=(S T)^{k+1} X .
$$

Also the forward implications are clear when (10.1) $S=T^{k}$ is a power of $T$. If instead we assume (10.2), then we argue

$$
(S T)^{-1}(0) \subseteq T^{-1}(0)+T(X) \text { and }(S T) X \supseteq T^{-1}(0) \cap T(X),
$$

while if $(U, V)$ satisfies $(10.2)$, then for arbitrary $k \in \mathbf{N}$

$$
V_{k} S^{k}-T^{k} U_{k}=I \text { with }\left\{U_{k}, V_{k}\right\} \subseteq \operatorname{comm}\left(S^{k}, T^{k}\right) .
$$

To verify (10.5) argue

$$
\begin{gathered}
S T x=0 \Longrightarrow x+T U x=V S x \in T^{-1}(0) ; \\
T(T x)=0 \Longrightarrow T x=T V S x-T U T x=(S T)(V x) .
\end{gathered}
$$

For (10.6) note that for arbitrary $k \in \mathbf{N}$

$$
V S-T U=I \Longrightarrow V^{k+1} S^{k+1}-T U\left(I+V S+\ldots+V^{k} S^{k}\right)=I .
$$

For an induced "spectrum" to be a closed set we have

11. Theorem. $T \in B L(X, X)$ is of finite descent; then so is $T-\lambda I$ for sufficiently small $\lambda \in \mathbf{C}$. 
Proof. This has been shown on Hilbert space by Han, Larson, and Pan ([13, Lemma 2.2 , Theorem 2.4). It is clear from the open mapping theorem (applied to condition (4.4) with $W=\{0\}$ ) that if condition (1.4) holds, then also

$$
S^{-1}(0)+(T-U)(X)=Y
$$

whenever $T-U \in B L(X, Y)$ is sufficiently close to $T \in B L(X, Y)$. The problem is that we must also perturb $S$. However if $S=T^{k}$ and $U=\lambda I$, so that $E_{X}(T-U)=$ $E_{X}(T)$, then we can argue

$$
E_{X}(T-U)+(T-U)(X)=E_{X}(T)+(T-U)(X) \supseteq S^{-1}(0)+(T-U)(X)=X .
$$

The subspaces of Definition 8 lead to certain special kinds of operators:

12. Definition. We shall call $T \in B L(X, X)$ triangular if the subspace $E_{X}(T)$ is dense:

$$
\operatorname{cl} E_{X}(T)=X \text {. }
$$

Dually $T \in B L(X, X)$ is co-triangular if the subspace $F_{X}(T)$ is trivial:

$$
F_{X}(T)=\{0\} .
$$

The shifts of Example 7 are either triangular or co-triangular:

13. Example. On each of the spaces $c_{0}$ and $\ell_{p}(1 \leq p<\infty)$, the forward shift $U$ is triangular, the backward shift $V$ is co-triangular and the standard weight $W$ is both triangular and co-triangular.

Proof. The hyperkernel of the backward shift is dense, since it includes all the "terminating" sequences:

$$
V^{-\infty}(0) \supseteq c_{00}
$$

Thus

$$
E(V) \supseteq V^{-\infty}(0) \text { is dense }
$$

and also

$$
F(V)=\bigcap_{|\lambda|=1}(V-\lambda I)^{\infty}(X) \supseteq \sum_{|\lambda|<1}(V-\lambda I)^{-\infty}(0) \supseteq V^{-\infty}(0) \text { is dense } .
$$

Since $U-\lambda I$ is one-to-one for every $\lambda \in \mathbf{C}$ we have

$$
E(U)=\{0\} \text { is trivial }
$$

and also

$$
F(U) \subseteq U^{\infty}(X)=\{0\} \text { is trivial } .
$$

Finally we notice that the weight $W$ commutes with the projection $U V$, and more generally

$$
W U^{n} V^{n}=U^{n} V^{n} W(n \in \mathbf{N})
$$

also for each $n \in \mathbf{N}$

$$
\begin{aligned}
& \left(\frac{1}{n} I-W\right)^{-1}(0)=U^{n-1}(I-U V) V^{n-1}(X), \\
& \left(\frac{1}{n} I-W\right)(X)=\left(U^{n-1}(I-U V) V^{n-1}\right)^{-1}(0),
\end{aligned}
$$

so that $E(W)$ is dense and $F(W)$ is trivial. 
Triangularity and Fredholmness co-operate to generate finite ascent or descent:

14. Theorem. If $T \in B L(X, X)$, then

$$
T \text { upper semi-Fredholm and co-triangular } \Longrightarrow T \text { of finite ascent }
$$

and

$$
T \text { lower semi-Fredholm and triangular } \Longrightarrow T \text { of finite descent. }
$$

Proof. If $T \in B L(X, X)$ is upper semi-Fredholm, then the finite ascent condition can be written in the form

$$
F_{X}(T) \cap T^{-1}(0)=\{0\} .
$$

Indeed since (8), Theorem 6.12.2) each power $T^{m}$ is also upper semi-Fredholm, then $T^{-m}(0)$ is finite dimensional for each $m \in \mathbf{N}$ and $T^{m}(X)$ is closed; thus if for each $m \in \mathbf{N}$ we have $T^{m}(X) \cap T^{-1}(0) \neq\{0\}$, then there is $\left(x_{m}\right)$ in $X$ for which

$$
\left\|T^{m}\left(x_{m}\right)\right\|=1 \text { and } T^{m+1} x_{m}=0 .
$$

By local compactness there is a subsequence

$$
\left(y_{m}\right)=T^{\phi(m)}\left(x_{\phi(m)}\right) \rightarrow y_{\infty} \in T^{\infty}(X),
$$

using the closedness of all the ranges, so that $\left\|y_{\infty}\right\|=1$ and $y_{\infty} \in F_{X}(T) \cap T^{-1}(0)$. This proves (14.1); towards (14.2) we claim that for subspaces $Y, Z \subseteq X$

(14.4) $Y$ closed of finite codimension and $Z$ dense $\Longrightarrow Y+Z=X$, because if $\operatorname{dim}(X / Y)=n$ find successively $e_{1}, e_{2}, \ldots, e_{n}$ with

$$
e_{j+1} \in Z \backslash\left(Y+\mathbf{C} e_{1}+\mathbf{C} e_{2}+\ldots+\mathbf{C} e_{j}\right) .
$$

Applying this with $Y=T(X)$ and $Z=E_{X}(T)$ gives (14.2).

It is clear that in (14.1) we can replace the "co-triangular" condition (12.2) by the weaker condition (14.3); dually in (14.2) we can replace the triangular condition (12.1) by the weaker condition

$$
\operatorname{cl} E_{X}(T)+T(X)=X .
$$

We cannot however remove the semi-Fredholm conditions: for example [12] if $T=$ $U \otimes V$ is the tensor product of the shifts, then (14.3) holds but we never get $T^{k}(X) \cap T^{-1}(0)=\{0\}$. For operators which are both upper semi-Fredholm and of finite ascent, or lower semi-Fredholm of finite descent ("semi Browder" in the sense of [8, Definition 7.9.1) the conditions of Theorem 10 can be replaced by simple commutivity (8], Theorem 7.9.2).

\section{REFERENCES}

[1] J. Anderson, On normal derivations, Proc. Amer. Math. Soc. 38 (1973) 136-140. MR 47:875

[2] M.R. Embry, Factorization of operators on Banach spaces, Proc. Amer. Math. Soc. 38 (1973) 587-590. MR 47:849

[3] C.-K. Fong, Normal operators on Banach spaces, Glasgow Math. Jour. 20 (1979) 163-168. MR 80e: 47020

[4] S. Grabiner, Generalizations of Fredholm operators, Banach algebras '97 pp 170-187, de Gruyter 1998. MR 99i:47022

[5] R.E. Harte, Berberian-Quigley and the ghost of a spectral mapping theorem, Proc. Royal Irish Acad. 78A (1978) 63-68. MR 80b:47004

[6] R.E. Harte, Almost open mappings between normed spaces, Proc. Amer. Math. Soc. 90 (1984) 243-249. MR 85b:47024 
[7] R.E. Harte, Almost exactness between normed spaces, Proc. Amer. Math. Soc. 100 (1987) 257-265. MR 88g:47004

[8] R.E. Harte, Invertibility and singularity, Dekker, New York 1988.

[9] R.E. Harte, Eine Kleine Gapmusik, PanAmerican Jour. 2 (1992) 101-102. MR 92k:47003

[10] R.E. Harte, Taylor exactness and Kaplansky's lemma, Jour. Op. Th. 25 (1991) 399-416. MR 94d:47002

[11] R.E. Harte, Exactness plus skew exactness equals invertibility, Proc. Royal Irish Acad. 97A (1997) 15-18. MR 99h:46085

[12] R.E. Harte, Variations on a theme of Kaplansky, Matematicki Vesnik (to appear).

[13] D. Han, D.R. Larson and Z. Pan, The triangular extension spectrum and algebraic extensions of operators, preprint.

[14] D.A. Herrero, D.R. Larson and W.R. Wogen, Semitriangular operators, Houston Jour. Math. 17 (1991) 477-499. MR 92m:47037]

[15] A.M. Sinclair, Eigenvalues on the boundary of the numerical range, Pacific Jour. Math. 35 (1970) 231-234. MR 42:5086

[16] A. Turnsek, Elementary operators and orthogonality, Linear Alg. Anal. 317 (2000) 207-216. MR 2002c: 47081

[17] K.W. Yang, Index of Fredholm operators, Proc. Amer. Math. Soc. 41 (1973) 329-330. MR 47:7492

School of Mathematics, Trinity College, Dublin 2, Ireland

E-mail address: rharte@maths.tcd.ie

Department of Mathematics, Texas A \& M University, College Station, Texas 778433368

E-mail address: larson@math.tamu.edu 